\title{
Flatus vaginalis a distressing symptom
}

\author{
Sabeena Allahdin
}

Accepted: 16 January 2011 /Published online: 29 January 2011

(C) Springer-Verlag 2011

\section{Dear Editor:}

Flatus vaginalis is the expulsion of trapped air from the vagina it is a natural phenomenon that occurs when air is forced into the vagina, and then expelled as the muscles of the vaginal wall naturally contract. Although air expelled from the vagina is usually harmless and odourless the sound of flatus vaginalis can be embarrassing and distressing for women who suffer from the condition. Vaginal flatus commonly occurs during or immediately after sexual intercourse. There are some cases in which vaginal flatulence may indicate a more serious medical condition; however, such as a rectovaginal fistula or female genital prolapse and it can occur postoperatively after vaginal surgery.

We report an unusual case of a young 17 year old nulliparous woman who presented with the compliant of flatus vaginalis on movement. She was greatly distressed by this symptom which had undermined her confidence and made her socially isolated. The flatus was completely odourless and she had no other symptom. Her BMI was 19 abdominal and vaginal examination showed no significant finding, she had a well supported uterus and the vaginal walls. One to one physiotherapy with pelvic floor exercises to increase the pelvic floor tone did not result in significant reduction in the patient's symptoms. The option of vaginal pessaries were not possible as even the smallest sized pessary was too big for her. We advised the use of simple tampons which resolved her symptoms completely. The tampon prevented air entry in to the vagina; they were easy to use and allowed the women more control of her symptoms allowing her to lead a more regular life style.

No disclosures
S. Allahdin $(\bowtie)$

St Mary's Hospital,

Newport Isle of Wight, UK

e-mail: drallahdin@hotmail.co.uk 